\title{
Research on Geographical Positioning of Ancient Map
}

\author{
Lili Jiang*
}

State Key Laboratory of Resources and Environmental Information System, Institute of Geographical Sciences and Natural Resources Research,, Chinese Academy of Sciences, Beijing 100101, China, jiang11@igsnrr.ac.cn

* Corresponding author

Keywords: Old maps, Ancient Map, Atlas, Geographical Positioning

\begin{abstract}
:
The ancient map has a large span of history, diverse types and rich content. Therefore, comprehensive analysis and summarization of ancient maps and geographical location in different categories are conducive to obtaining more ideal geographical correction results. How to classify the ancient map classification suitable for geographical correction, and then carry out targeted geographic coordinate correction is the primary research content of this research. The geographic reference and geographic reference of the map are the main factors determining the geographical location of the map. Therefore, the division of the ancient map according to the presence or absence of georeferencing is a prerequisite for solving the difficulty of correcting the ancient map.

The geographic parameters of ancient maps mainly include map projection, geographic reference, map accuracy, scale and so on. In the study of ancient map geolocation, the first thing to be solved is the determination of ancient map projection and geo-reference. For maps with different mathematical foundations (projection and geo-reference), different positioning methods are used for geo-correction. Only by determining the mathematical basis, projection and geographic reference of the ancient maps that need to be located, can the corresponding projection conversion method or geographic correction method be used for map positioning. However, the mathematical basis of ancient maps is not clearly marked on the map, and even if it is marked, it is often not very accurate. Therefore, it is necessary to study the acquisition methods of mathematical parameters of ancient maps.
\end{abstract}

For different ancient canal maps, different methods are needed to determine the basis of their geolocation:

(1) Latitude and longitude

The latitude and longitude survey of the ancient canal map is based on the modern Western measurement method, through projection conversion, and the latitude and longitude map on the map. The latitude and longitude measurement map has a clear geographic reference and projection.

(2) Similar to modern latitude and longitude

This type of map does not indicate the age of production, cartographers and geo-references, or incorrectly label cartographers, geo-references or geographic benchmarks, but according to the presence or absence of latitude and longitude and latitude and longitude, the map's performance techniques, drawing characteristics, related content, etc. In addition to the reference frame of the latitude and longitude network, some maps also have geo-references for the grid, which can be used to determine whether such maps are modern latitude and longitude georeferences. There is a gap between the accuracy of such maps and the measured maps.

(3) Grid in the square

Grid in the square is an important traditional Chinese method for mapping maps. It uses a grid coordinate system of square squares. It is an auxiliary line for drawing maps on an ancient scale. The length of each square is the number of real points. Quite a scale of today's maps. The map drawn by the method of "counting in the painting" is more accurate than the predecessors and is reliable. According to this method, the map has been used for more than 500 years (from the drawing time of the trace map). According to records, this method began with the principle of "drawing six bodies" proposed by China's Jin Dynasty. The "six bodies" are the "scores", which is the current scale; the second is the "preview", which is used to determine the mutual orientation of the landforms and features; and the third is the "daoli" to determine the road between the two places. The distance is four; the fourth is "high"; the fifth is "Fang", that is, the fluctuation of the slope of the ground; the sixth is "straight", that is, the conversion of the height of the field and the distance on the map. This is a milestone in the history of maps in China. Because such maps are greatly improved in accuracy, the role in multidisciplinary fields is worth paying attention to. Moreover, such maps are often compiled with reference to a certain map, such as Huayi map, trace map, map of Yu, map of the Emperor, map of the emperor's work, and the preparation of the records of the history of the party, all based on the previous one. of. Therefore, when correcting, you can classify them into one category and consider them together. 


\section{(4) Landscape imagery}

The image of the ancient canal of the landscape image painting adopts the expression of "the law of the landscape", that is, all kinds of ground elements on both sides of the river are drawn toward the center line of the river. This type of map, because of the "reality" is very strong, the mountains and rivers are realistic and rich in color, so it has always been the mainstream painting method of the ancient rivers before the Ming and Qing Dynasties. There are many types of maps, large quantities, rich map content, and extremely high historical value, but their compilation is very different from modern maps. First, the coordinate directions in ancient maps are "upper south and north", and also " "Upper north and lower south", mainly "upper south and north", which is different from the directional principle of the modern map "up north and south"; secondly, the use of visually intuitive painting to express features, and the use of less map symbols There is a certain proportional relationship between the positional relationship between the features, but it is quite different from the modern maps with strict mathematical foundations; 